\title{
Stiffness analysis of corrugated laminates under large deformation
}

\author{
C. Thurnherr*, L. Ruppen, S. Brändli, C.M. Franceschi, G. Kress, P. Ermanni \\ Laboratory of Composite Materials and Adaptive Structures, Department of Mechanical and Process Engineering, ETH Zürich, Tannenstr. 3, CH-8092 Zürich, Switzerland
}

\section{A R T I C L E I N F O}

Article history:

Received 10 June 2016

Revised 17 October 2016

Accepted 19 October 2016

Available online 21 October 2016

Keywords:

Corrugated laminates

Non-linearity

Flexible skins

Anisotropy

\begin{abstract}
A B S T R A C T
This paper presents a detailed investigation about the geometric non-linear stiffness behavior of corrugated laminates in six different load cases. The considered tensile, bending, and shear load cases allow the modeling with a unit-cell approach assuming a generalized plane strain state. The torsional load case is more complex. There the mechanical response depends on the number of unit-cells and the width of the samples in case of geometric non-linearities. We first identify the load cases that show a non-linear stiffness response under large deformation. Then the non-linear behavior is analyzed in detail using numerical simulation and we aim for mechanical explanations to describe the non-linear behavior. The FE simulations of the torsional load case are validated using experiments with 3D printed samples.
\end{abstract}

(c) 2016 Elsevier Ltd. All rights reserved.

\section{Introduction}

Corrugated structures have a highly anisotropic behavior due to their geometry. They have a very high compliance in one direction while they have very high bending stiffness about the same axis due to the significant amount of material placed away from the neutral axis. The high bending stiffness is achieved with relatively low material use, this makes them very attractive for lightweight structures for example in cardboards [1] or as structural elements in aircrafts e.g in Junkers Ju-52 to increase the stability [2]. Furthermore, they were suggested as flexible skins [3] for morphing wing applications [4-6] or lately also for artificial muscles [7]. Recently, Dayyani et al. published a review paper about corrugated structures in morphing aircrafts [8]. Due to their geometry, corrugated structures can undergo large deformations while the local strains remain relatively low [9]. In many applications the material behavior remains linear, but the large global deformations can cause non-linearities. Hence, it is important to analyze the geometric non-linear behavior of these structures.

Several models exist to calculate the initial stiffness response of corrugated laminates in the form of equivalent plate properties. Xia et al. $[10,11]$ suggested a model to calculate the homogenized equivalent stiffness values for thin and balanced laminates. Briassoulis et al. [12] modeled the equivalent mechanical properties of thin orthotropic corrugated plates. Kress et al. suggested a closed-form solution for thin corrugated laminates consisting of circular sections [13] and a numerical model that is also valid for

\footnotetext{
* Corresponding author.

E-mail address: thclaudi@ethz.ch (C. Thurnherr).
}

thick laminates [14]. Biancolini et al. [1] presented a model to calculate the equivalent stiffness properties for corrugated boards. Dayyani et al. [15] investigated equivalent models for composite corrugated cores coated with an elastomer. All these models are only valid for linear analysis and do not take into account geometric non-linearities that occur under large deformation.

There also exist some investigations about the non-linear behavior of corrugated laminates. Liew et al. [16] and Peng et al. [17] presented studies about the non-linear mechanical response of corrugated skins based on the mesh-free Galerkin method and first-order shear deformation theory. Thill et al. [18] investigated the mechanical behavior of trapezoidal shaped corrugated laminates under large tensile displacements transverse to the corrugation direction. Dayyani et al. [19] conducted a numerical and experimental study about the behavior of composite corrugated cores. Lately, we [20] presented an analytical model, validated with experimental and numerical data, that can predict the non-linear stiffness response of corrugated laminates in tensile loading. A comprehensive study on the non-linear structural response of corrugated laminates under all possible singly applied static loading situation can as of yet not be found in literature.

The present paper presents an investigation about the geometric non-linear behavior of corrugated structures and studies the mechanics that drive the non-linear stiffness response. We consider six load cases, in particular two tensile load cases, two bending load cases, a shear load case and a torsion load case. The authors are aware of the fact that the principle of superposition does not hold in the case of non-linear analysis. Hence, we would like to emphasize that the study aims to understand the non-linear mechanisms in corrugated laminates in different loading rather 
than presenting an equivalent stiffness model where various loadings can be coupled.

\section{Modeling of corrugated laminates}

This section presents the load cases that are considered, shows the definition of the geometry and the coordinate systems, and it introduces the notation of the constitutive stiffness matrix of corrugated laminates. Then the used numerical models are described, namely a unit-cell and a full model, and at the end of the section the material properties are listed.

Fig. 1 shows the six load cases that describe the mechanical response of corrugated sheets. Although in the non-linear case it is not valid to superpose the different loadings, a detailed analysis of the non-linear behavior of each of the six load cases gives important insights about the mechanism that have to be considered if dealing with geometric non-linearities. In this paper we only consider geometric non-linearities (and non-linear contacts in load case 5), but we assume that the material behavior remains linear.

We consider corrugated shapes consisting of circular sections defined as shown in Fig. 2. The parameters are the amplitude $c$, the laminate thickness $t_{\text {lam }}$, the radius of the circular section $R$, the periodic cell-length $p$ and the sample width $w$ in the $x$-direction. We use a global coordinate system $x, y$ and $z$ and a local coordinate system $s, t$.

To denote the single stiffness terms of a flat laminate, we use the well-known notation of the classical laminate theory where the ABD-matrix links the strains and curvatures with line forces and moments [21]:

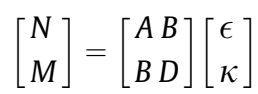

To indicate the stiffness terms of the corrugated laminate we use the superscript $\sim$

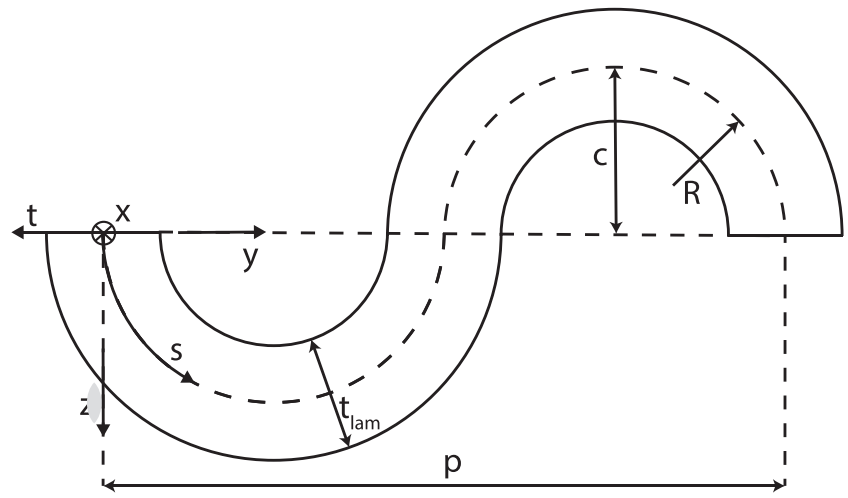

Fig. 2. Corrugated structures consisting of circular sections.

$\left[\begin{array}{l}N \\ M\end{array}\right]=\left[\begin{array}{ll}\widetilde{A} & \widetilde{B} \\ \widetilde{B} & \widetilde{D}\end{array}\right]\left[\begin{array}{l}\epsilon \\ \kappa\end{array}\right]$

In order to reduce the numerical effort, we can take advantage of the periodicity of corrugated structures by only modeling one unit-cell. Further, we can also consider a generalized plane strain state in the $x$-direction. Fig. 3 shows two possible unit-cells. The red one is mostly used when modeling corrugated structures; we also use this unit-cell for load case $1-4$. However, for bending about the $x$-axis in load case 5 we found out that the green unitcell is more advantageous if contact between the unit-cells occurs. In this case the green unit-cell allows to model self-contact without introducing rigid walls. Certainly, the results are independent of the choice of the unit-cell. For the twist load-case the unit-cell and generalized plane strain modeling is not as straight forward for large deformations. Furthermore, this load-case should be compared to experiments where the samples cannot fulfill an infinite width. Therefore, we use a full model with four unit-cells and a
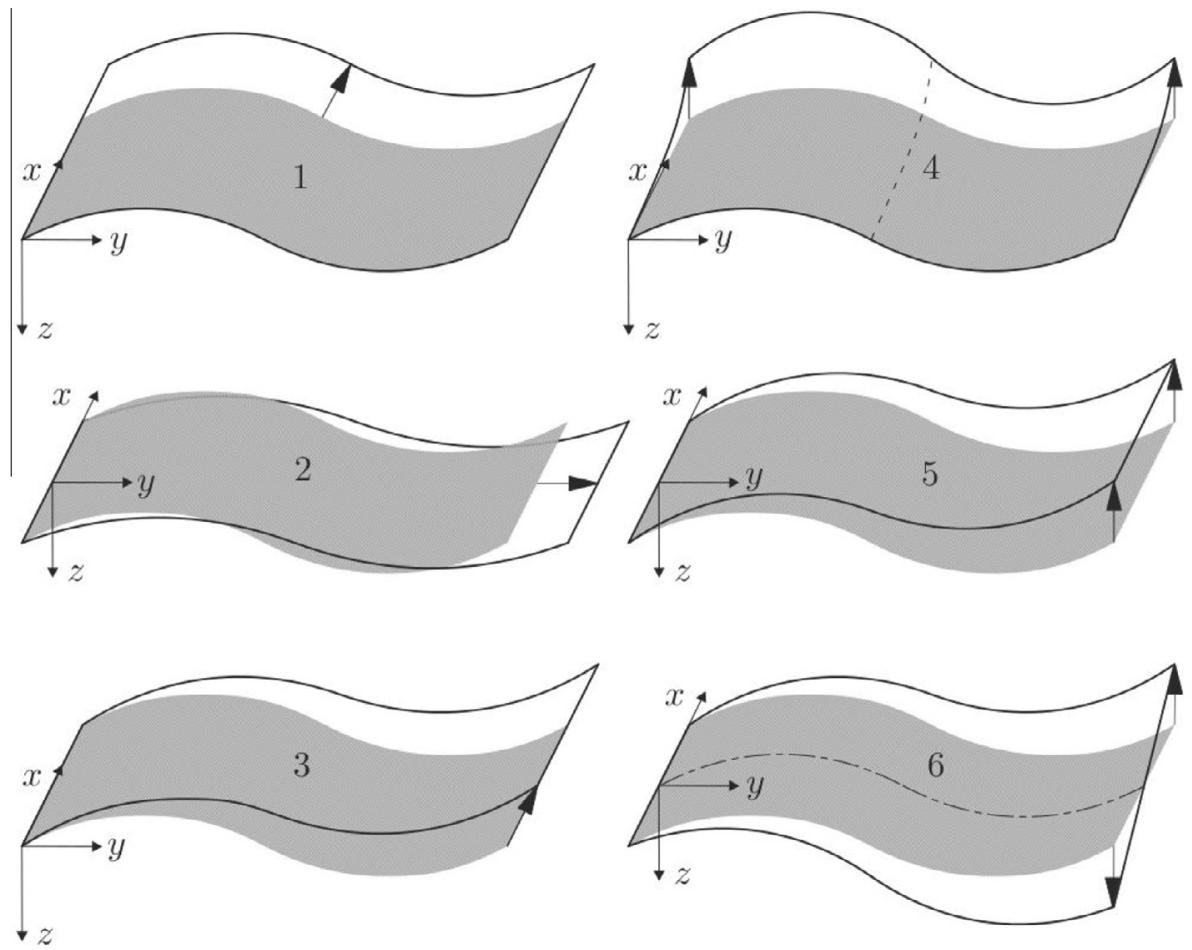

Fig. 1. Load cases [13]. 


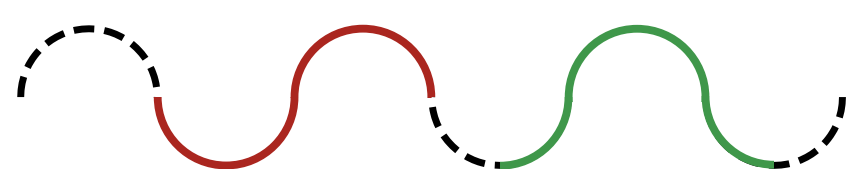

Fig. 3. Possible unit-cells (red and green).

finite width in the present study to investigate the twist load case. The authors are aware of the fact that the results of a model with a finite width will be influenced by free edge effects.

\subsection{Unit-cell model}

For all considered load cases except the torsion load case we can use a unit-cell model. We implement two different unit-cell models as shown in Fig. 4. All simulations are conducted with Ansys Mechanical classic 14.5. The first model (see Fig. 4a) is a 3D model consisting of SOLID186 elements with 16 nodes for load case 1,2,3, and 5 and 20 nodes (including mid-node in the $x$-direction) for load case 4 . We use one element per layer through the thickness, 40 elements along the $s$-direction, and one element in the $x$-direction. One element per layer through the thickness is sufficient to obtain accurate stiffness results in which we are interested in this study since the studied laminate is very thin. The stress results might be less accurate, however, we only use them for qualitative illustration of the different load cases in the present paper. In the $x$ direction we assume a generalized plane strain state which means that the distribution of all state variables is homogeneous along $x$. On both edges of the unit-cell we apply periodic boundary conditions. The unit-cell has an amplitude $c$ of $20 \mathrm{~mm}$ and a periodic cell length $p$ of $100 \mathrm{~mm}$. The model consist of 2 layers. The model that is used for all the calculations is shown in Fig. 4a (i). Since the model consists of only two layers, it is very thin and it is difficult to visualize stress distributions with colors. Therefore, we scale the thickness and the width in the post-processing (as shown in ii)) to plot stress distributions, the quantitative stress values remain the same in the post-processing.

For the bending about the $x$-axis non-linear contact can occur if the corrugation amplitudes are very large. Therefore, we introduce a second unit-cell model as shown in Fig. 4b. Since in this load case there are no enforced displacements in the $x$-direction, and the

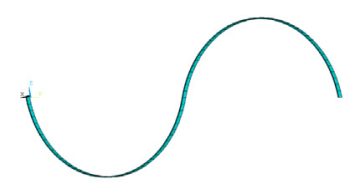

i) Model for the FE simulation

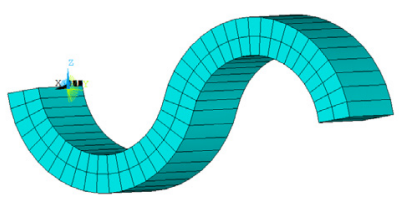

ii) Post-processed model with scaled thickness (40x) to visualize stress distribution

a) 3D unit-cell model

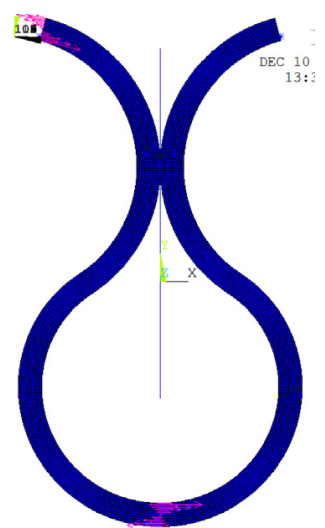

b) $2 \mathrm{D}$ unit-cell model to model self-contact
Fig. 4. Unit-cell model: (a) 3D unit-cell which allows to model displacements in direction of the generalized plane strain (i) how it is used for the FE calculations and (ii) a post-processed model with scaled thickness to be able to visualize the stress distributions and (b) 2D unit-cell model that can be used to model self-contact in load case 5 . strains along $x$ may be assumed to be very small, we can reduce the model to a 2D model with a special plane-strain state. We use the PLANE183 element with 8 nodes. Also here we use one element per layer, and, considering that we need a finer mesh due to the contact and the fact that the arc length is longer for corrugations with large amplitudes, 400 elements along the s-direction. The contact was modeled with contact elements of the type CONTA172 using the penalty method. The geometry is defined with an amplitude of $c=80 \mathrm{~mm}$ and a periodic cell length $p=100 \mathrm{~mm}$.

\subsection{Full model}

For the torsion load case a generalized plane strain exists along convected coordinates which circumstance cannot be mapped with our unit-cell model in Ansys Mechanical. Also a 2D unit-cell model with plane strain elements is not suitable since it does not allow loading in the $x$-direction. Furthermore, the FE simulations should be validated with experiments. Hence we need a FE model that has the same geometry and boundary conditions as in the experiments. Since the samples have a finite width, the mechanical response is influenced by free edge-effects and generalized plane strain does not hold anymore. Therefore, we use a full model for load case 6 as illustrated in Fig. 5. The model consists of four unit cells and a finite width. We test different geometries. Since we also verify these simulations with experiments and the test samples are printed with a 3D printer, the dimensions of the samples have to be chosen according to their manufacturability. We chose the following geometric parameters: $p=40 \mathrm{~mm}=$ const, $c=[5 \mathrm{~mm}$, $10 \mathrm{~mm}, 30 \mathrm{~mm}$ ], and the width is $25 \mathrm{~mm}$ and $50 \mathrm{~mm}$, respectively.

\subsection{Materials}

To model the corrugated laminates, we used a carbon fiber reinforced plastic (CFRP) namely a high modulus carbon fiber epoxy composite. Its properties are listed in Table 1 . We tested two different lay-ups: a $[0,0]^{\circ}$ and $\mathrm{a}[90,90]^{\circ}$ configuration. Each layer has a thickness of $0.125 \mathrm{~mm}$.

To fabricate the test samples for the experiments we used a $3 \mathrm{D}$ printer and all specimens were made from PolyLactic acid (PLA). Its properties are shown in Table 2 . In order to be able to compare the numerical and experimental results we used PLA for the simulation with the full model. The samples made from PLA have a thickness of $0.52 \mathrm{~mm}, 0.55 \mathrm{~mm}$, and $0.975 \mathrm{~mm}$ for amplitudes of $c=5 \mathrm{~mm}, c=10 \mathrm{~mm}$, and $c=30 \mathrm{~mm}$, respectively. The different thicknesses of the different geometries are due to the fabrication process. We measured a tolerance in the thickness of about $\pm 0.05 \mathrm{~mm}$.

\section{Geometric non-linearities}

In the following subsections the results of the FE simulations are presented for the different load cases. The stiffness response for large deformations is analyzed and the load cases where

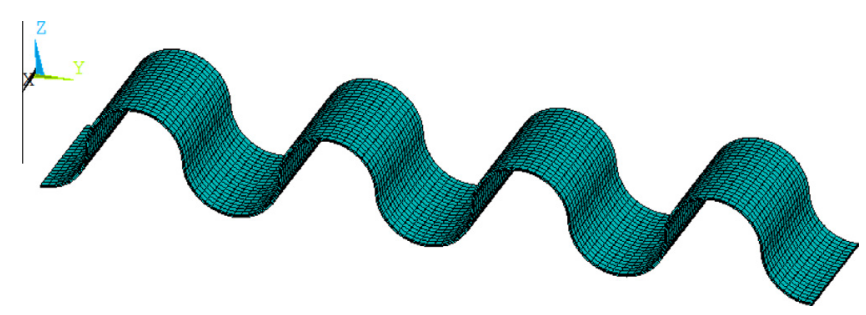

Fig. 5. FEM model with four unit-cells and a finite width. 
Table 1

Material properties of high modulus Carbon Fibre Epoxy Composite.

\begin{tabular}{|c|c|c|c|c|c|}
\hline$E_{11}[\mathrm{GPa}]$ & 290 & $E_{22}[\mathrm{GPa}]$ & 5 & $E_{33}[\mathrm{GPa}]$ & 5 \\
\hline$G_{12}[\mathrm{GPa}]$ & 5 & $G_{13}[\mathrm{GPa}]$ & 5 & $G_{23}[\mathrm{GPa}]$ & 2.083 \\
\hline$v_{12}[-]$ & 0.41 & $v_{13}[-]$ & 0.41 & $v_{23}[-]$ & 0.2 \\
\hline
\end{tabular}

Table 2

Material properties of PLA

\begin{tabular}{ll}
\hline$E_{11}[\mathrm{GPa}]$ & 3.5 \\
$G_{23}[\mathrm{GPa}]$ & 1.3 \\
$v_{23}[-]$ & 0.346
\end{tabular}

geometric non-linearities occur are identified. For the torsion load case we present a detailed study including experimental validation.

The enforced loads are chosen in a way that the material strength is not exceeded. The limit is set to a maximum material strain of $1 \%$. Hence geometric non-linearities are only investigated in the range before material failure is expected. Since the maximum allowable material strain for PLA is higher than for a typical fiber reinforced plastic material, the PLA-samples for the experiments can reach higher local strains. For the simulation, however, material non-linearities are not considered.

Within all the colored deformation plots of the 3D unit-cell model for load case $1-5$, the thickness is scaled to have a better visibility of the stress distributions.

Within this paper, we do not combine the non-linear stiffness response with the stiffness of the underlying base sheet laminate, since such comparisons between initial base and corrugated sheet stiffness has been shown by other authors [13].

\subsection{Load case 1}

In load case $1 \mathrm{a}$ global strain $\epsilon_{x}$ is enforced in $x$-direction. One unit-cell is modeled using periodic boundary conditions and generalized plane strain. The results are obtained using CFRP material. Fig. 6 shows the deformed shape for load case 1 . The resulting stiffness response depends on the cross-section area and the normal force in $x$-direction. Therefore, it is obvious that we will not observe geometric non-linearities which is confirmed looking at Fig. 7. This figure shows the stiffness component $\widetilde{A}_{11}$ normalized with the initial stiffness.

\subsection{Load case 2}

Load case 2 is a tensile load case where a global strain $\epsilon_{y}$ is enforced in $y$-direction. The results are obtained using CFRP

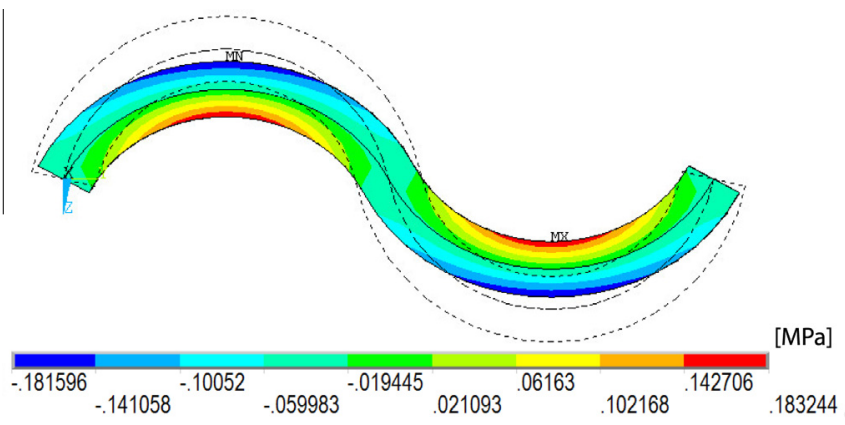

Fig. 6. Deformation in load case 1 , the colors represent the stress distribution $\sigma_{y}$ the deformations are scaled by a factor of 50 . (For interpretation of the references to colour in this figure caption, the reader is referred to the web version of this article.)

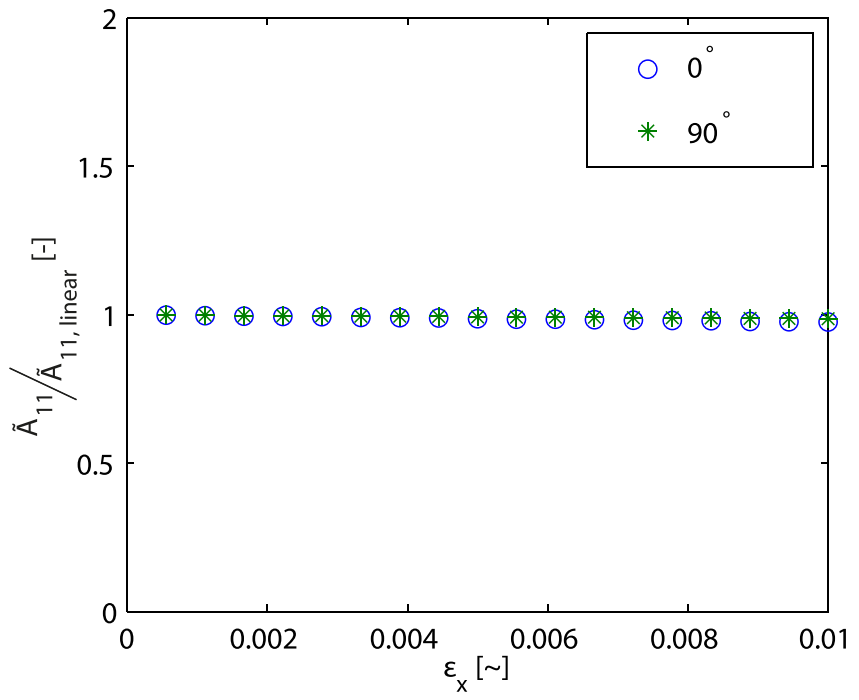

Fig. 7. Normalized stiffness $\widetilde{A}_{11}$ for different global strains $\epsilon_{x}$.

material. In Fig. 8 we see the deformed unit-cell. Fig. 9 shows the normalized stiffness values $\widetilde{A}_{22}$ for different $\epsilon_{y}$. The stiffness depends on the enforced global strain, hence the mechanical response is highly non-linear.

We extensively discussed the geometric non-linear response of this load case in a recently submitted paper [20]. Understanding the governing mechanism we suggested an analytical model and verified it with simulations and experiments using different geometries. The non-linear stiffness response can be explained with the governing mechanism shown in Fig. 10. The mechanical response is driven by the bending moment $M$ and the normal force $N$. In the beginning, when the amplitude is large, the response is mainly driven by the moment $M$. The more the corrugated structure deforms, the smaller the amplitude gets. Hence, the moment gets less and the normal force more important. This results in the typical stiffness response as illustrated in Fig. 11.

\subsection{Load case 3}

In load case 3 a shear strain $\epsilon_{x y}$ is enforced. The results are obtained using CFRP material. Fig. 12 illustrates the deformed unit-cell in the shear load case and Fig. 13 shows the normalized
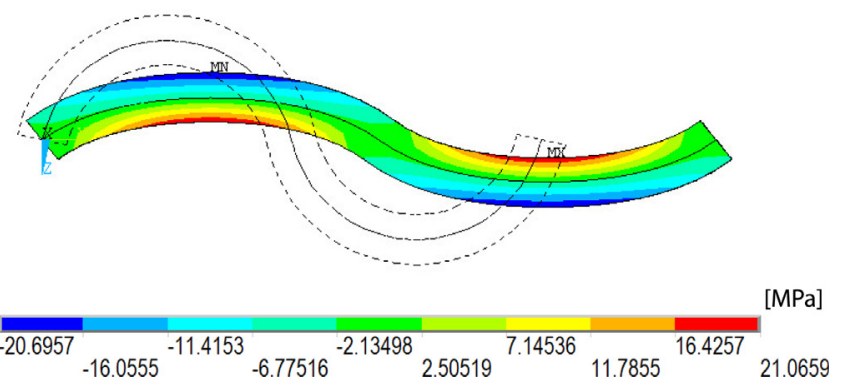

Fig. 8. Deformation in load case 2, the colors represent the stress distribution $\sigma_{y}$. 


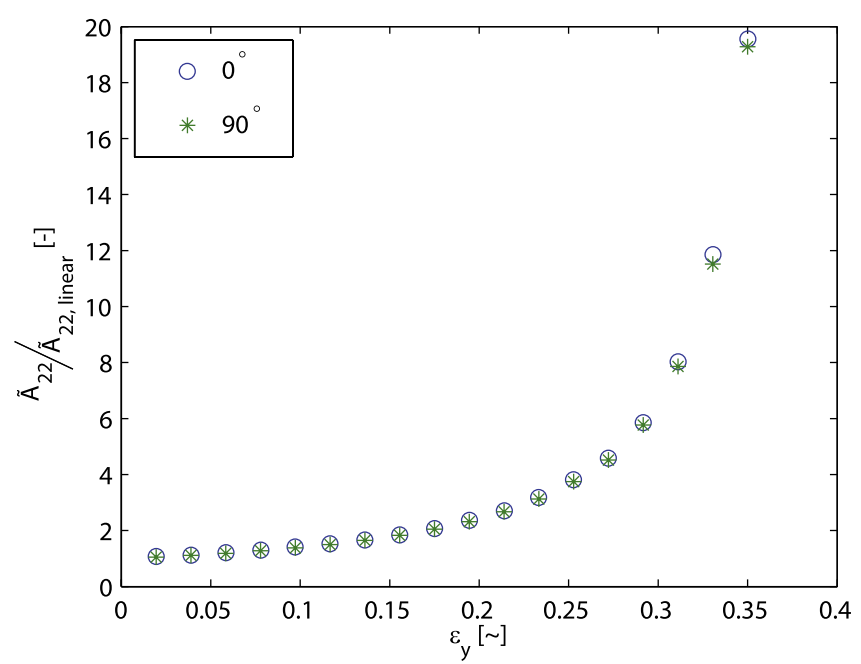

Fig. 9. Normalized stiffness $\widetilde{A}_{22}$ for different global strains $\epsilon_{y}$.

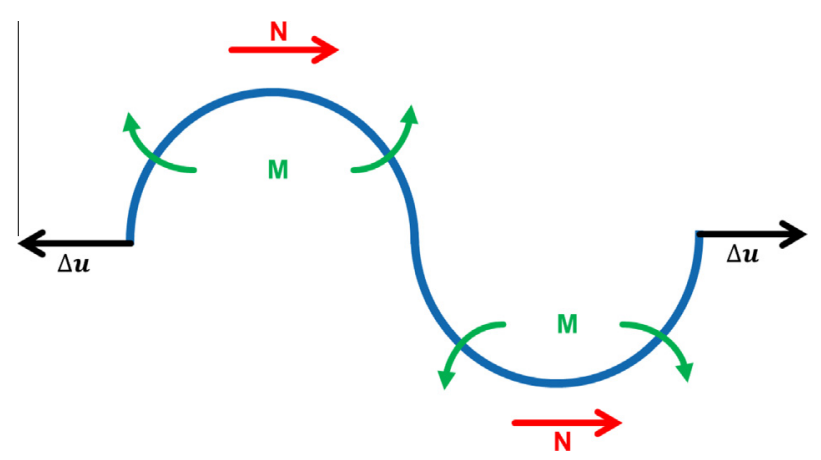

Fig. 10. Governing mechanism for tensile loading in $y$-direction [20].

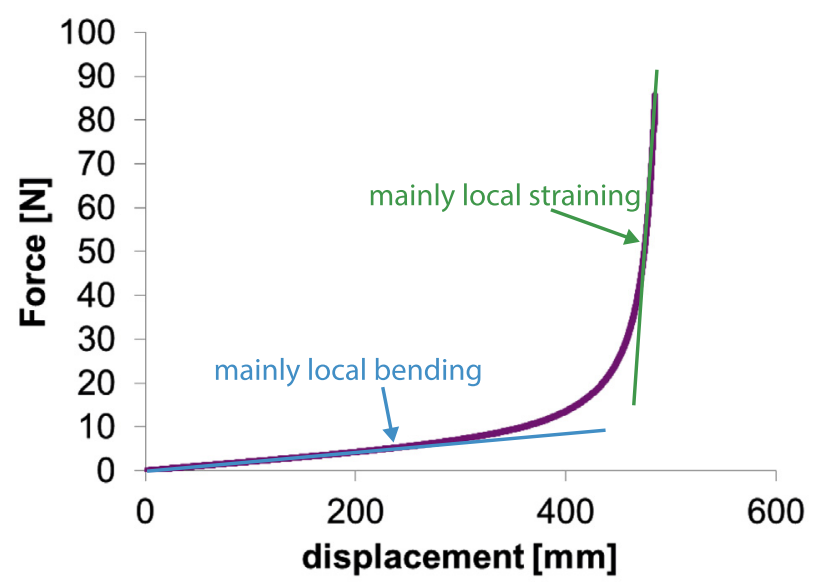

Fig. 11. Typical stiffness response for tensile loading in $y$-direction: in the beginning the stiffness depends on the bending stiffness, and in the end on the axial sheet stiffness [20].

stiffness $\widetilde{A}_{66}$ for different shear strains. As we can see from the plot the stiffness value does not change with increasing strains, since the governing mechanisms remain the same.

\subsection{Load case 4}

Load case 4 describes a bending about the global $y$-axis as shown in Fig. 14. The results are obtained using CFRP material.

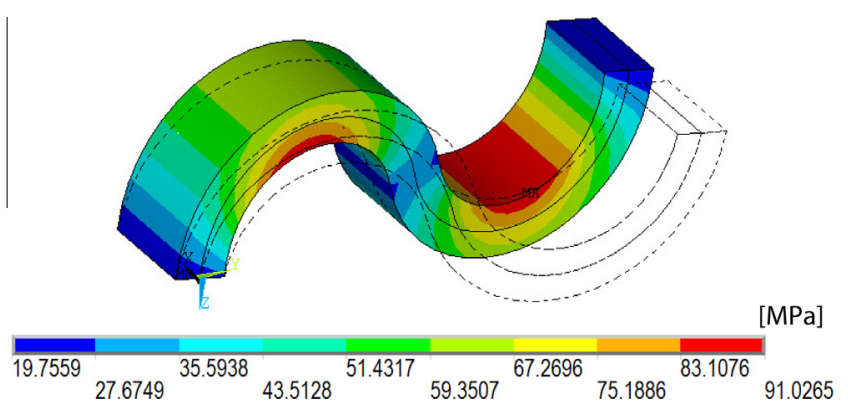

Fig. 12. Deformation in load case 3 , the colors represent the stress distribution $\tau_{x y}$, the deformation is scaled by a factor of 10 . (For interpretation of the references to colour in this figure caption, the reader is referred to the web version of this article.)

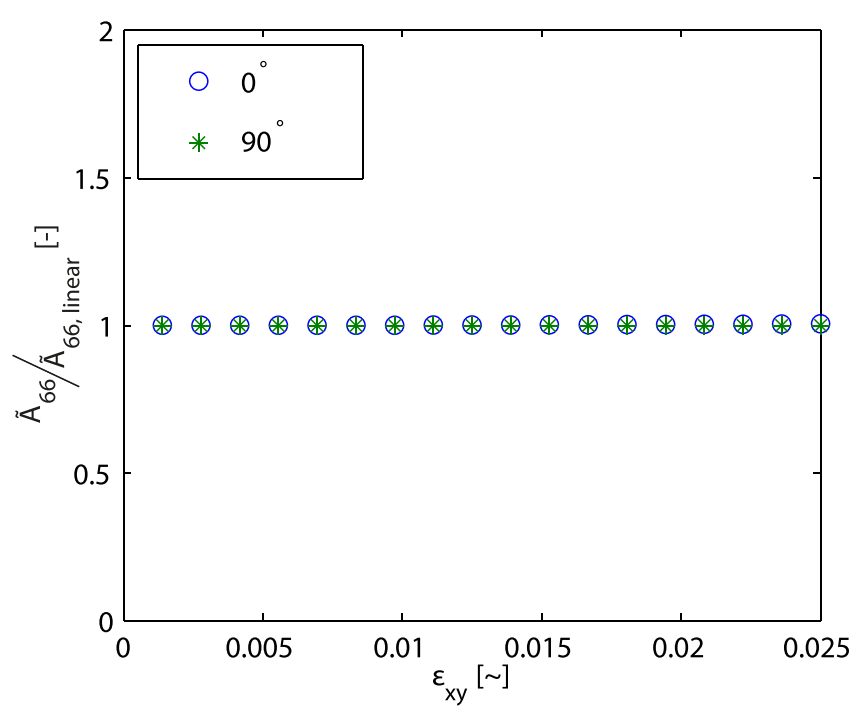

Fig. 13. Normalized stiffness $\widetilde{A}_{66}$ for different global shear strains $\epsilon_{x y}$.

To investigate this load case we use a unit-cell with periodic boundary conditions and apply a generalized plane strain state. The stiffness response for different curvatures $\kappa_{x}$ is illustrated in Fig. 15. The stiffness values $\widetilde{D}_{11}$ are normalized with the initial stiffness. For deformations up to the point where the maximum material strain is reached, the bending stiffness keeps its value since the governing mechanisms remain the same.

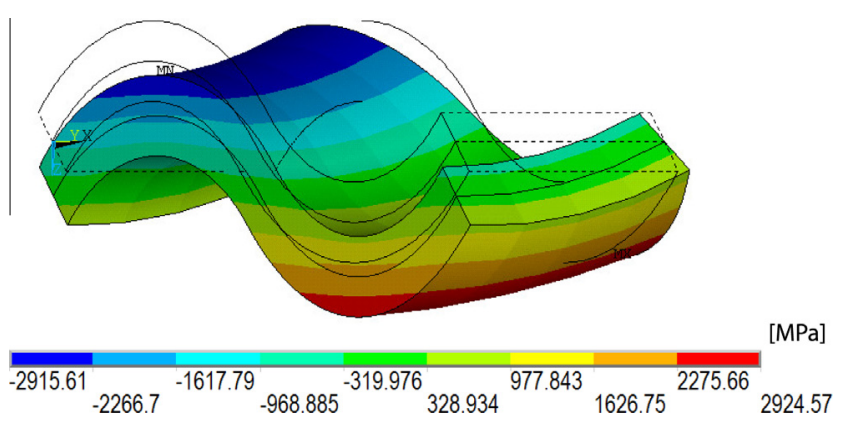

Fig. 14. Deformation in load case 4 , the colors represent the stress distribution $\sigma_{x}$. (For interpretation of the references to colour in this figure caption, the reader is referred to the web version of this article.) 


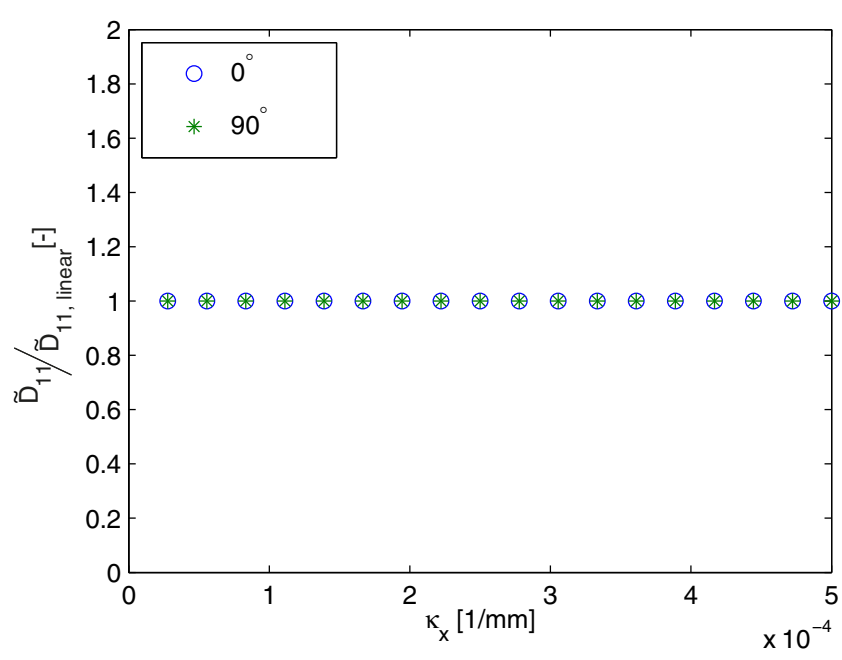

Fig. 15. Normalized stiffness $\widetilde{D}_{11}$ for different global curvature $\kappa_{x}$.

\subsection{Load case 5}

In load case 5 we investigate a bending about the global $x$-axis as illustrated in Fig. 16. The unit-cell model is used and periodic boundary conditions are applied and generalized plane strain is assumed. The results are obtained using CFRP material. If the unit-cells do not touch each other, the bending stiffnesses $\widetilde{D}_{12}$ and $\widetilde{D}_{22}$ remain constant also for large enforced curvatures $\kappa_{x y}$ and $\kappa_{y}$, respectively, as we can see in Fig. 17.

If the amplitudes are large, self-contact occurs between the unit-cells if the structure is bent about the $x$-axis. This selfcontact increases the bending stiffness as illustrated in Fig. 18. Hence, the bending stiffness response becomes non-linear resulting in a moment-curvature curve with characteristic tangent stiffness without and with contact.

\subsection{Load case 6}

For the torsion load case the unit-cell assumption does not hold anymore in case of geometric non-linearity in the global coordinate system. This can easily be explained with Fig. 19: while the line in the rotation center keeps its length (blue) all the lines at $x \neq 0$ change their length (green to red). Therefore, the evaluation of an equivalent non-linear stiffness is not possible, instead the non-linear stiffness curves of different finite samples are shown in the following.

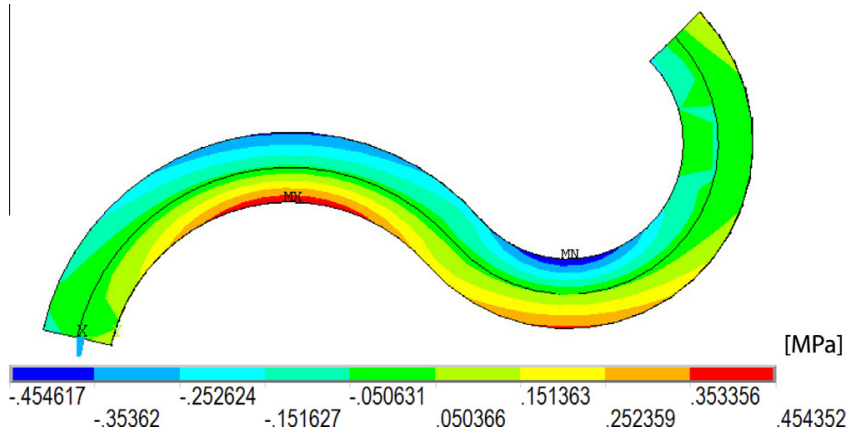

Fig. 16. Deformation in load case 5 , the colors represent the stress distribution $\sigma_{y}$. (For interpretation of the references to colour in this figure caption, the reader is referred to the web version of this article.)

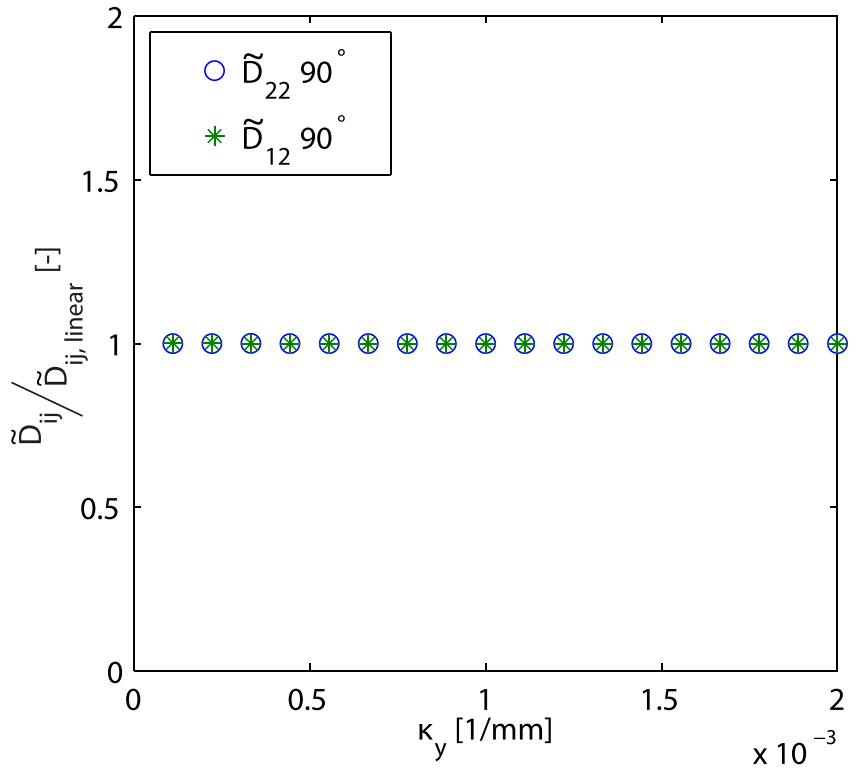

Fig. 17. Normalized stiffness $\widetilde{D}_{12}$ and $\widetilde{D}_{22}$ for different global curvature $\kappa_{x y}$ and $\kappa_{y}$, respectively.

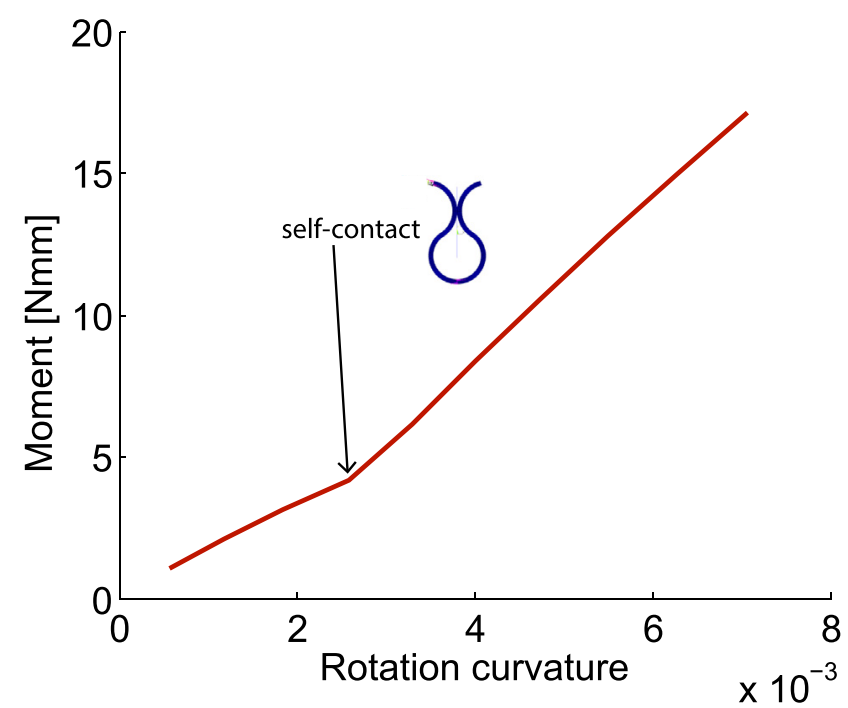

Fig. 18. Typical moment-curvature curve for corrugations with large amplitudes: after self-contact of the unit-cells the bending stiffness increases.

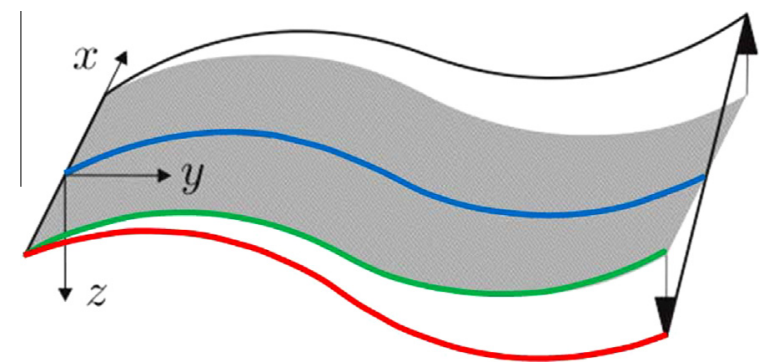

Fig. 19. In case of twist the generalized plane strain assumption does not hold anymore for large deformations in the global coordinate system: while the line in the rotation center keeps its length (blue) the arc length changes for $x \neq 0$ (green to red). (For interpretation of the references to colour in this figure caption, the reader is referred to the web version of this article.) 
Therefore, this load case is studied with a full FE model containing four unit-cells and a finite width. The numerical results are verified with experimental data. The results are obtained using PLA material. For large twists the corrugation tends to get unstable. The FE simulation does only cover the region before these instabilities occur. The experiments show the stability behavior containing several instabilities for some samples.

Six different geometries are tested with different amplitudes and widths. The periodic cell length is $40 \mathrm{~mm}$, the amplitudes are $c=5,10$ and $30 \mathrm{~mm}$. The width $w$ is $25 \mathrm{~mm}$ and $50 \mathrm{~mm}$. The samples are made from PLA using a 3D printer.

\subsubsection{Experiments}

Fig. 20 shows the six different samples that were tested. To test the samples on a tensile test machine we designed a test set-up where we are able to transform the traverse displacement into a rotation and the measured reaction force into a torque. The test set-up is shown in Fig. 21. One side is clamped while the other side is mounted on balanced alloy wheel that can rotate. Two ball bearings guarantee that the friction onto the wheel is minimized. Then the force is redirected to a second wheel with a carbon roving and then to the traverse of the tensile machine. Also this wheel is mounted on a ball bearing. For the tests we use a Zwick tensile testing machine with a load cell of $5 k N$. The rotation is calculated by multiplying the traverse displacement by the radius of the clamping wheel. A degree template is glued onto the wheel to double-check the values. The torque is calculated by multiplying the measured force by the radius of the wheel. The tests were

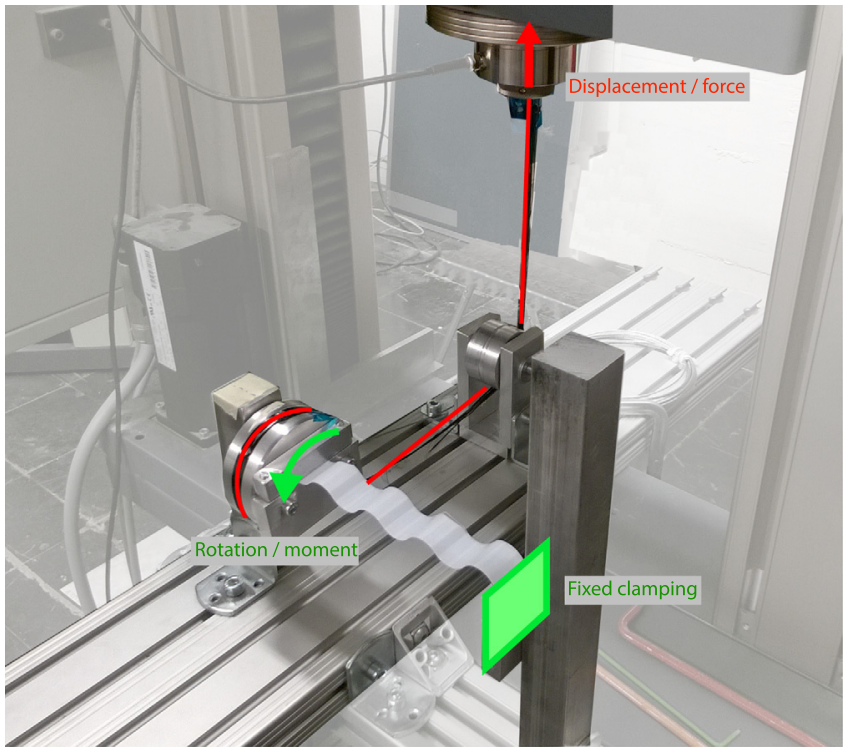

Fig. 21. Experimental test-setup for the torsional load case.

conducted at low speed, each experiment taking about three minutes to be completed. The test temperature was around $22^{\circ}$. The samples were tested shortly after printing them, hence the liquid
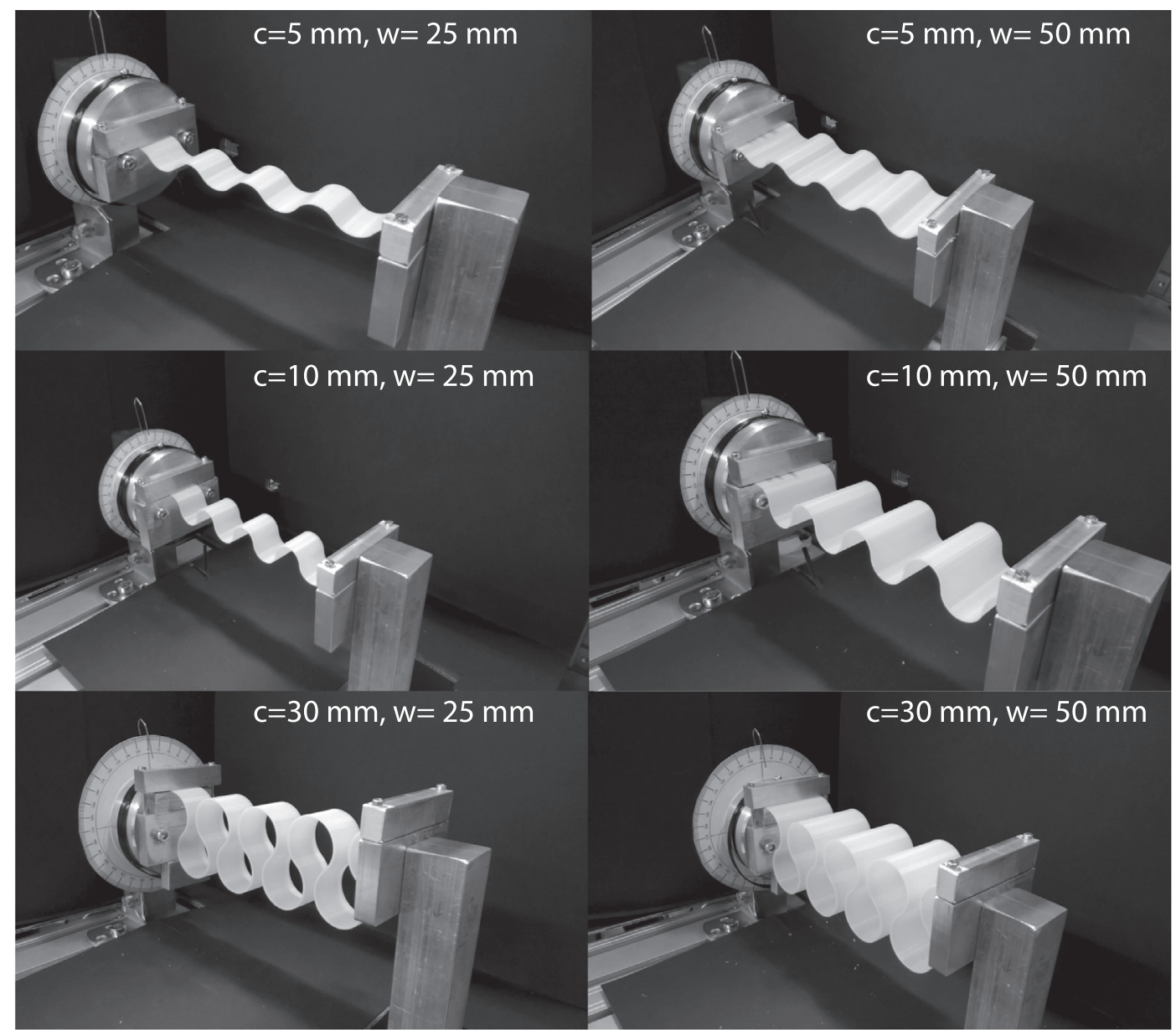

Fig. 20. Tested samples mounted in the test bench. 
concentration can be assumed to be very low. According to [22] PLA is a very stable material and the creeping strains remain lower than $0.05 \%$ for our operating temperatures and liquid concentrations. Therefore, creeping was not considered in the study.

Four cameras are used to visually capture the experiment. One camera is positioned on top of the experiment, one in front, one takes pictures from the side, and one tracks the degree template.

\subsubsection{Comparison between numerical and experimental results}

In this section we first show a qualitative comparison between the numerical and experimental results and analyze the deformation behavior for the different samples. Then we present a quantitative comparison, namely the torque-rotation-curves, and comment the different stiffness responses.

In all the following figures, the colors in the numerical results denote the displacement in $y$-direction. For the same amplitude the deformed shapes qualitatively look similar for both tested widths. Therefore, we only show illustrations of the deformed shapes for the wider samples since they correspond more with a corrugated plate rather than a beam.

Fig. 22 shows the deformed shapes observed in the experiments and simulation for specific rotations for the samples with an amplitude of $5 \mathrm{~mm}$ and a width of $50 \mathrm{~mm}$. We can see that the numerical and experimental results agree very well. With increasing rotation the corrugations straighten at the free edges. For rotation angles of $250^{\circ}$ and higher we observe plastic deformations which are not considered in the simulation.

Fig. 23 shows the deformation plots for an amplitude of $10 \mathrm{~mm}$ and a width of $50 \mathrm{~mm}$. We observe a different deformation behavior than for the samples with the lower amplitudes due to the longer arc-length. As the rotation angle increases, first the free edges also start to straighten, but at a rotation of $410^{\circ}$ for the wider sample, the corrugation gets unstable and collapses. We observe a second instability at a rotation of $680^{\circ}$ for $w=25 \mathrm{~mm}$ and $555^{\circ}$ for $w=50 \mathrm{~mm}$, respectively.

Fig. 24 shows the deformed shapes for the samples with an amplitude of $30 \mathrm{~mm}$ and a width of $50 \mathrm{~mm}$. For both widths the global behavior is similar. At a rotation angle of $220^{\circ}$ for $w=50 \mathrm{~mm}$ self-contact between the unit-cells occurs. Since we do not consider non-linear contact in the FE simulation, we stop the simulation at this point. If the rotation is further increased, we observe again an unstable behavior and the corrugation collapses. At $315^{\circ}$ for $w=50 \mathrm{~mm}$, the corrugated structures touches the ground of the experimental test set-up and the experiment is stopped.
In the following part of this subsection, the quantitative comparison between the numerical and experimental results is presented by means of the torque-rotation-curves.

Fig. 25 shows the comparison between experimental and numerical data for the first configuration with an amplitude of $5 \mathrm{~mm}$. We can see that the experiments are very robust and repeatable. For a width of $w=25 \mathrm{~mm}$ the samples fail after a rotation of about $520^{\circ}$. The experimental and numerical results agree very well up to a rotation angle of $320^{\circ}$, after that the FEM simulation predicts experimentally measured stiffness too high. At this point we also observed plastic deformation during the experiments which explains the difference to the simulation. Also for $w=50 \mathrm{~mm}$ the FE simulation matches very well with the experiments. Again the simulation gets stiffer than the experiments at a rotation of about $160^{\circ}$, this can be explained with the fact that the non-linear material behavior is not considered in the FE model. The sample with a width of $50 \mathrm{~mm}$ is stiffer than the one with a width of $25 \mathrm{~mm}$ as expected. The overall behavior remains the same for both widths. A non-linearity due to large deformation can clearly be identified.

Fig. 26 shows the experimental and numerical results for an amplitude of $10 \mathrm{~mm}$. All the experimental samples show a robust behavior. In the experiment we could see two instabilities that can clearly be identified in the torque-rotation curve as a drastic stiffness loss. These instabilities lead to a non-linear behavior. Again the FEM simulation is able to reproduce the experiments in the area before the stiffness loss appears. Some deviations occur, due to the linear material model in the FE simulation. Nonlinearities due to large rotations can clearly be identified in the stiffness curve. As expected, the wider sample is stiffer, the global behavior remains the same.

Fig. 27 shows the results for the samples with an amplitude of $30 \mathrm{~mm}$. The tested samples are robust and show the same behavior. For a width of $w=25 \mathrm{~mm}$ the stiffness up to a rotation of about $200^{\circ}$ remains almost linear with a slight increasing slope for higher rotations. Between $200^{\circ}$ and $300^{\circ}$ the torque stays almost constant and then it rapidly decreases. The experiments were stopped at the point where the corrugation started to touch the ground of the experimental set-up. In comparison to the experiments with the lower amplitudes, non-linear material behavior was not observed for these samples. The simulation delivers results up to $200^{\circ}$ and reproduces the experimentally found behavior very well. For a width of $w=50 \mathrm{~mm}$ the global behavior is similar to the experiments with $c=30 \mathrm{~mm}$ and $w=25 \mathrm{~mm}$, however, the samples are stiffer. The experiments are repeatable, non-linear material

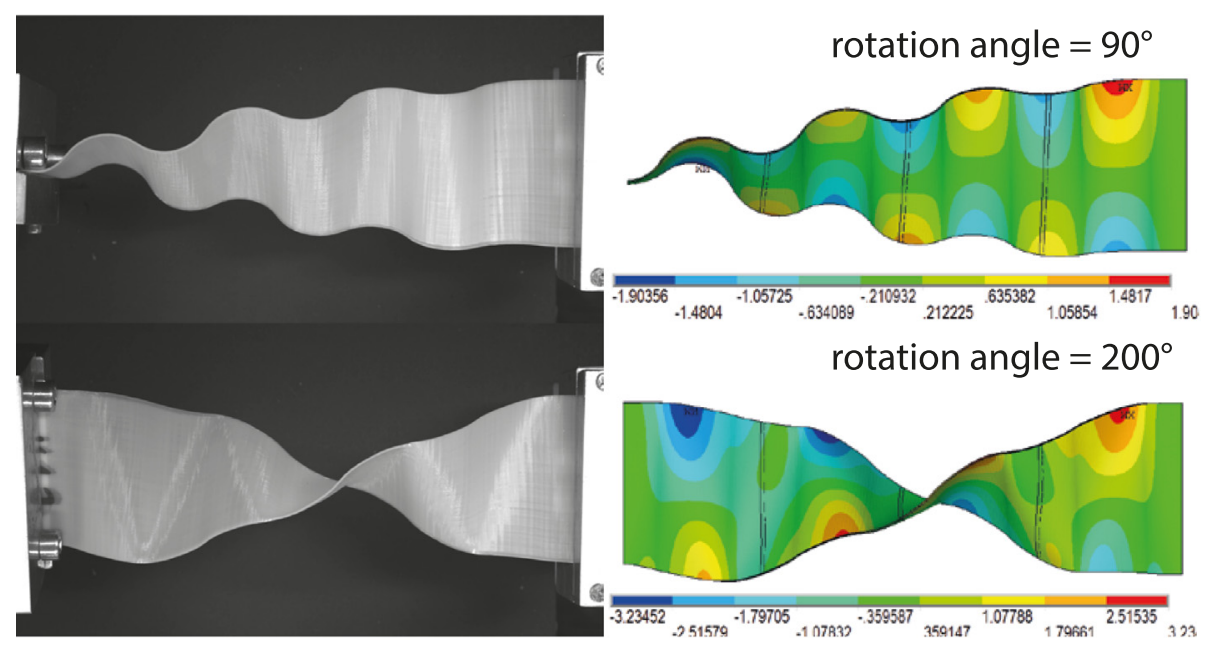

Fig. 22. Visualization of numerical and experimental results for $c=5 \mathrm{~mm}$ and $w=50 \mathrm{~mm}$. 

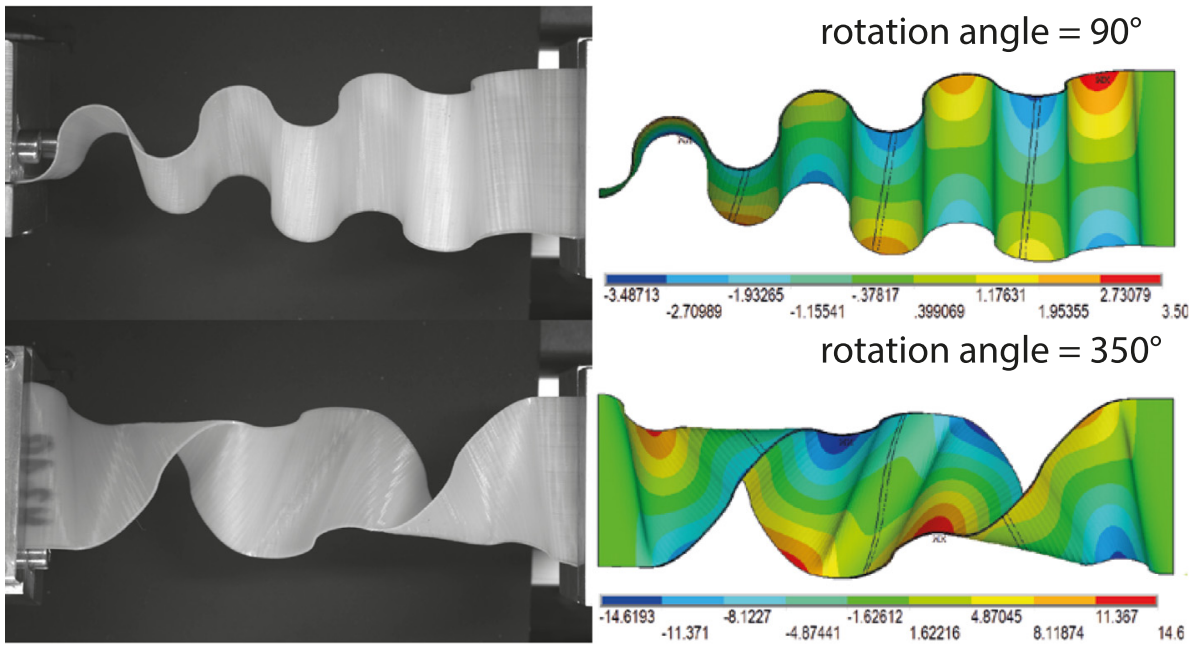

Fig. 23. Visualization of numerical and experimental results for $c=10 \mathrm{~mm}$ and $w=50 \mathrm{~mm}$.
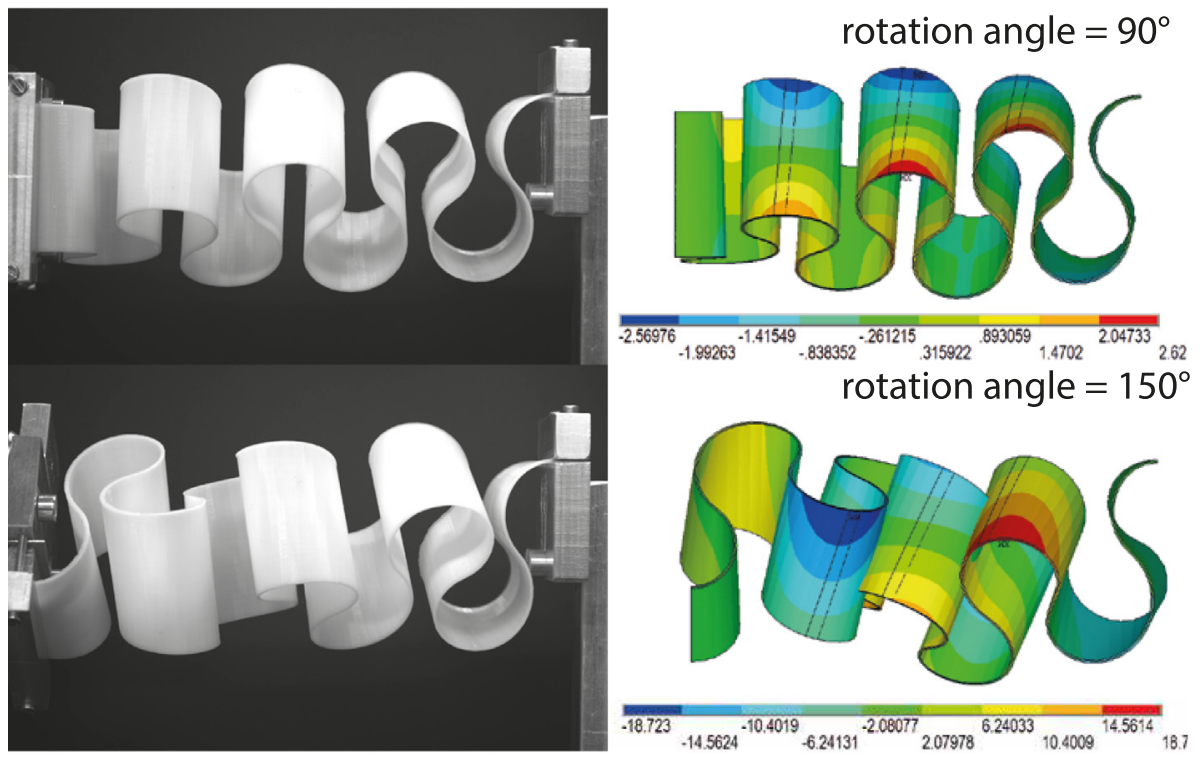

Fig. 24. Visualization of numerical and experimental results for $c=30 \mathrm{~mm}$ and $w=50 \mathrm{~mm}$.

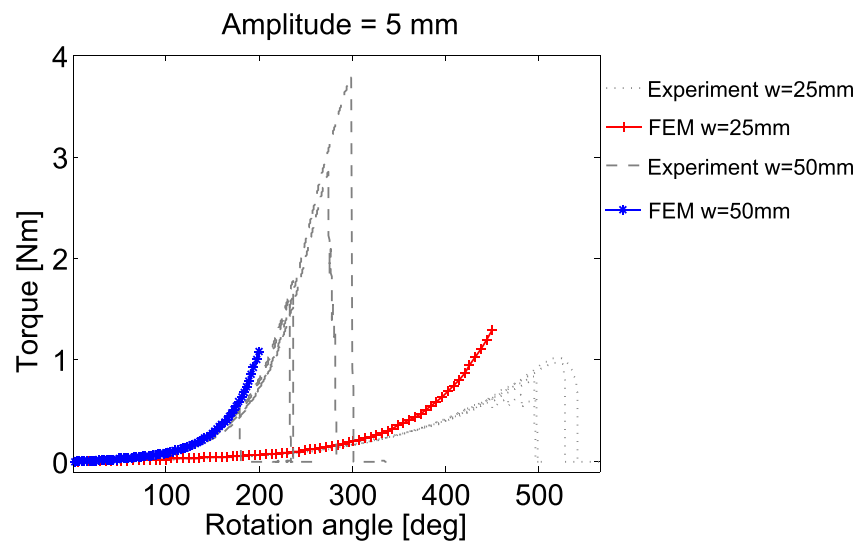

Fig. 25. Comparison between numerical and experimental results for $c=5 \mathrm{~mm}$.

behavior was not observed. The simulation agrees very well with the experimental results.

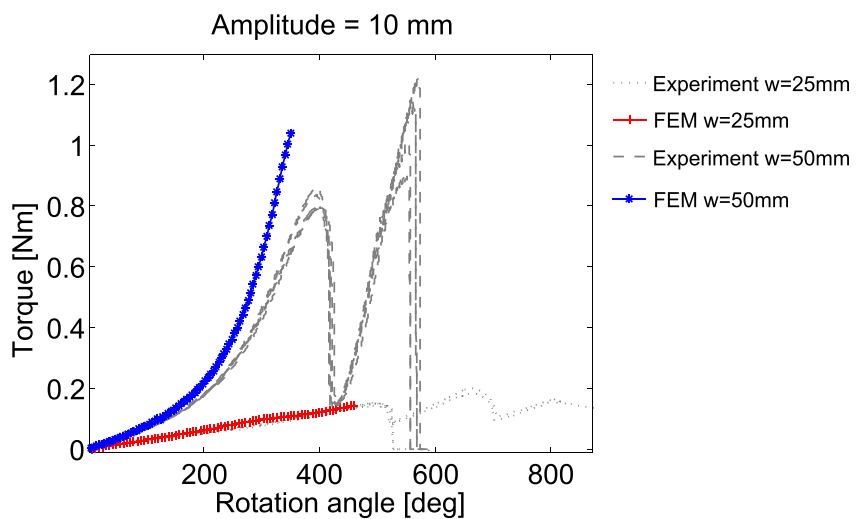

Fig. 26. Comparison between numerical and experimental results for $c=10 \mathrm{~mm}$.

For all the samples we observed some differences between the experiments and the simulations. These can mainly be explained with the fact that the simulation does not take into account 


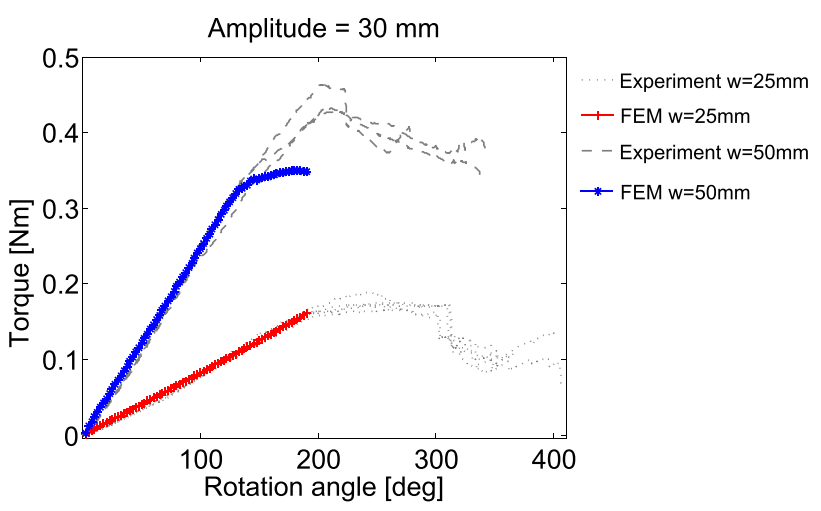

Fig. 27. Comparison between numerical and experimental results for $c=30 \mathrm{~mm}$.

material non-linearity and non-linear contact. Further reasons might be the tolerances in the thickness and the periodic-cell length of the fabricated samples, uncertainties in the material data, and the fact that the simulation assumes isotropic material behavior.

\section{Discussion}

Experiments as well as simulations show that the twist deformation of load case 6 leads to structural response with different characteristics, dependent on corrugation amplitude. In particular, specimens with large corrugation amplitude buckle at critical twist whereas those with smaller corrugation amplitude deform in a stable manner until material failure occurs. The phenomenon results from two competing mechanisms. The inherently stabilizing mechanism is illustrated with Fig. 28, where it is shown that a twisted prismatic body, whose ends are kept at the same distance, experiences direct strains constant along the original straight lines which have become helix-shaped. The direct strain increases with increasing distance from the center of rotation and creates positive direct stress whose resultant of the crosssectional area gives a tensile force, preventing instability. This straightening effect dominates the specimens with small corrugation amplitude, but ceases to be effective at higher amplitudes, where the arc-length along the corrugations is much larger than the distance between the clamps.

The remaining question, how the stiffness response is influenced, if coupling of the different loading is considered, will be addressed in future studies.
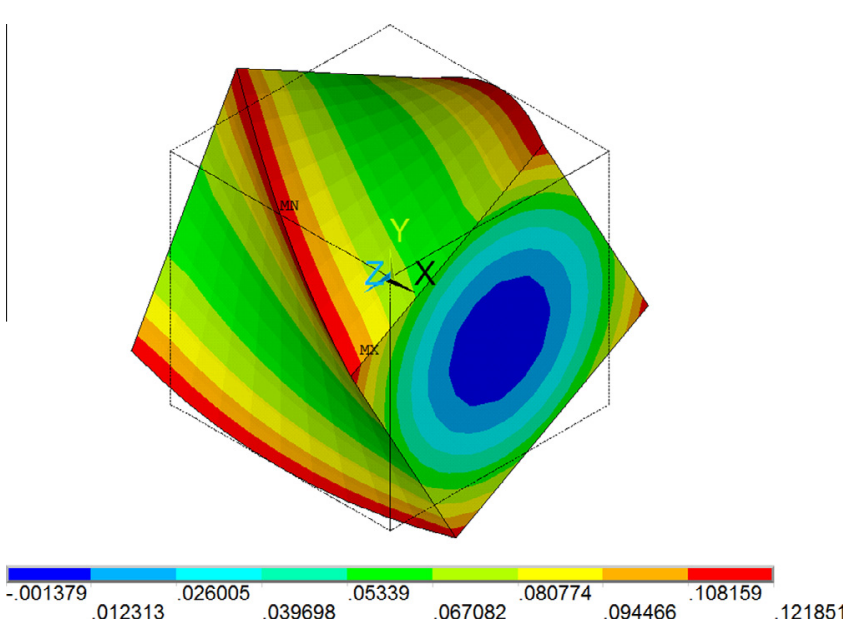

Fig. 28. Illustration of the strains in $x$-direction in case of torsional loading.

\section{Conclusion}

In the present paper we investigated the geometric non-linear behavior of corrugated laminates in six different loading situations. In load case 1,3 , and 4 we found that material strength is exceeded before geometric non-linearities can occur. All the non-linear stiffness curves show a very progressive behavior with a knee where the stiffness changes. For load case 2, tensile loading in $y$ direction, the stiffness response is highly non-linear due to large deformations. In load case 5 , bending about the $x$-axis, the stiffness remains linear until self-contact between the unit-cells occur. At this point the stiffness increases. In load case 6 , the large deformations also resulted in geometric non-linearities. Due to its complexity load case 6 was investigated numerically and experimentally. The experiments are very robust and reproducible and agree very well with the FE simulation. We observed that the qualitative non-linear stiffness response mainly depends on the corrugation amplitude.

These results can be used in design processes where large deformations might occur, such as morphing wings, to estimate whether linear modeling is sufficient or geometric non-linearities have to be considered. We can conclude that non-linearities have to be taken into account for structural materials in load case 2 and 6, and in load case 5 if self-contact occurs. To increase the applicability for design purposes, we plan to model combinations of the different load cases and conduct parametric studies in future work.

\section{Acknowledgment}

The authors gratefully acknowledge the support of the Swiss National Science Foundation (project No. 149200 and Grant No. 206021_150729).

\section{References}

[1] Biancolini M. Evaluation of equivalent stiffness properties of corrugated board. Compos Struct 2005;69(3):322-8. doi: http://dx.doi.org/10.1016/ j.compstruct.2004.07.014.

[2] Hirschel E-H, Prem H, Madelung G, Bergmann JW. Luftfahrtforschung in Deutschland. Bernard \& Graefe Verlag; 2001.

[3] Yokozeki T, Takeda S-I, Ogasawara T, Ishikawa T. Mechanical properties of corrugated composites for candidate materials of flexible wing structures. Compos Part A: Appl Sci Manuf 2006;37(10):1578-86.

[4] Barbarino S, Bilgen O, Ajaj RM, Friswell MI, Inman DJ. A review of morphing aircraft. J Intell Mater Syst Struct 2011;22(9):823-77. doi: http://dx.doi.org/ 10.1177/1045389X11414084.

[5] Thill C, Etches JA, Bond IP, Potter KD, Weaver PM. Composite corrugated structures for morphing wing skin applications. Smart Mater Struct 2010;19 (12):124009. doi: http://dx.doi.org/10.1088/0964-1726/19/12/124009.

[6] Thill C, Etches J, Bond I, Potter K, Weaver P. Morphing skins. Aeronaut J 2008;112(3216):117-39.

[7] Kadooka K, Taya M, Naito K, Saito M. Modeling of a corrugated dielectric elastomer actuator for artificial muscle applications. Proc SPIE 2015;9430:943020-9430203. doi: http://dx.doi.org/10.1117/12.2084733.

[8] Dayyani I, Shaw AD, Saavedra Flores E, Friswell M. The mechanics of composite corrugated structures: a review with applications in morphing aircraft. Compos Struct 2015;133:358-80.

[9] Winkler M, Kress G. Deformation limits for corrugated cross-ply laminates. Compos Struct 2010;92(6):1458-68.

[10] Xia Y, Friswell MI, Equivalent Models of Corrugated Laminates for Morphing Skins, Active and Passive Smart Structures and Integrated Systems 7977 (2011) 79771I-79771I-10. doi:http://dx.doi.org/10.1117/12.880433.

[11] Xia Y, Friswell MI, Flores EIS. Equivalent models of corrugated panels. Int J Solids Struct 2012;49(13):1453-62.

[12] Briassoulis D. Equivalent orthotropic properties of corrugated sheets. Comput Struct $1986 ; 23: 129-38$.

[13] Kress G, Winkler M. Corrugated laminate homogenization model. Compos Struct 2010;92(3):795-810.

[14] Kress G, Winkler M. Corrugated laminate analysis: a generalized plane-strain problem. Compos Struct 2011;93(5):1493-504. doi: http://dx.doi.org/ 10.1016/i.compstruct.2010.12.004.

[15] Dayyani I, Friswell MI, Ziaei-Rad S, Saavedra Flores EI. Equivalent models of composite corrugated cores with elastomeric coatings for morphing structures. Compos Struct 2013;104:281-92. 
[16] Liew KM, Peng LX, Kitipornchai S. Nonlinear analysis of corrugated plates using a FSDT and a meshfree method. Comput Methods Appl Mech Eng 2007;196(21-24):2358-76. doi: http://dx.doi.org/10.1016/i.cma.2006.11.018.

[17] Peng LX, Liew KM, Kitipornchai S. Analysis of stiffened corrugated plates based on the FSDT via the mesh-free method. Int J Mech Sci 2007;49(3):364-78. doi: http://dx.doi.org/10.1016/j.ijmecsci.2006.08.018.

[18] Thill C, Etches JA, Bond IP, Potter KD, Weaver PM, Wisnom MR. Investigation of trapezoidal corrugated aramid/epoxy laminates under large tensile displacements transverse to the corrugation direction. Compos Part A: Appl Sci Manuf 2010;41(1):168-76. doi: http://dx.doi.org/10.1016/ i.compositesa.2009.10.004.
[19] Dayyani I, Ziaei-Rad S, Salehi H. Numerical and experimental investigations on mechanical behavior of composite corrugated core. Appl Compos Mater 2011;19(3-4):705-21.

[20] Thurnherr C, Ruppen L, Kress G, Ermanni P. Non-linear stiffness response of corrugated laminates in tensile loading. Compos Struct 2016;157:244-55.

[21] Jones RM. Mechanics of composite materials. New York: Hemisphere Publishing Corporation; 1975.

[22] Widiastuti I, Sbarski I, Masood SH. Creep behavior of PLA-based biodegradable plastic exposed to a hydrocarbon liquid. J Appl Polym Sci 2013;127 (4):2654-60. doi: http://dx.doi.org/10.1002/app.37575. 